\title{
Fractional Slot Winding Design Method of Permanent-Magnet Synchronous Machines using Slot Star Diagram
}

\author{
Yasuhiro Miyamoto ${ }^{1 *}$, Tsuyoshi Higuchi ${ }^{2}$, Takashi Abe ${ }^{2}$, Yuichi Yokoi ${ }^{2}$ \\ ${ }^{1}$ Yaskawa Electric Corporation, Japan \\ ${ }^{2}$ Nagasaki University, Japan \\ E-mail:miya@yaskawa.co.jp \\ E-mail²: thiguchi@nagasaki-u.ac.jp
}

\begin{abstract}
In recent years, Permanent-Magnet-Type Synchronous Machine is used in many applications for industrial drive for the improved performance. Especially, for the armature winding, the fractional slot winding is known about the improved efficiency performance and torque ripple performance.

In this paper, we proposed the irregular pitch winding on the fractional slot winding to improve torque ripple characteristics, used the rectangle shape magnet. At the case of the conventional regular pitch winding, we can calculate the winding factor $K_{w}$ by the typical formula. But at this irregular pitch, it is too difficult to seek $K_{w}$ from the formula. So, we propose the solution to seek $K_{w}$ used "Slot Star Diagram" on the irregular pitch, and we seek the optimum the irregular pitch angle to decrease the harmonic component in EMF considered with the field magnet shape.

For it, we explain how to derive by using "Slot Star Diagram" for the winding arrangement to be simply. And in the case of the odd and even slot, it has given the examples for each, which describes the procedure of derivation of the winding factor $K_{w}$.
\end{abstract}

\section{INTRODUCTION}

The Fractional Slot Winding (later shows FSW) is called that its number of slots per pole per phase is fractional number. The winding arrangement of Integral Slot Winding (later shows ISW) is arranged phase $\mathrm{U}, \mathrm{V}$ and $\mathrm{W}$ coils regularly. But, FSW' arrangement is irregularly. Further, the number of slots of ISW is even only. On the other hand, the case of FSW has the both even and odd number. So, on the winding design of FSW, it is difficult to design used the same method at the case of ISW ${ }^{[1]}$. In addition, we considered about the irregular pitch winding to improve electromotive force (later shows EMF) performance of Permanent Magnet type Synchronous machine.

So, in this paper, we propose the winding design method used "Slot Star Diagram", for the FSW design. So far, Slot Star Diagram is introduced in a variety of literature. There we explained about the derivation of the winding factor used Slot Star Diagram for the irregular pitch winding. And we consider the optimum design between irregular pitch angle and gap flux distribution, to decrease harmonic components in EMF for torque ripple reduction

\section{TyPe OF Fractional SLOT Winding METHOD ${ }^{[1]}$}

Table1 shows the example of FSW. FSW is separated 3 type of method. They are " $q<1 / 2$ ", " $1 / 2<q<1$ " and " $1<q$ ", they all has the both even and odd number of slot showed on Tabel1. The number of even slots is designed by 10 poles, and odd is designed by 8poles.
Table1. Type of Fractional Slot Winding Method

\begin{tabular}{|c|c|c|c|c|c|c|}
\hline FSW & \multicolumn{2}{|c|}{$\begin{array}{l}q<1 / 2 \\
\text { Concentrated } \\
\end{array}$} & \multicolumn{2}{|l|}{\begin{tabular}{|l|}
$1 / 2<q<1$ \\
Distributed
\end{tabular}} & \multicolumn{2}{|c|}{$\begin{array}{l}1<q \\
\text { Distributed }\end{array}$} \\
\hline Slot & Even & Odd & Even & Odd & Even & Odd \\
\hline \multirow[t]{2}{*}{$q=a+c / b$} & $\mathrm{~A}$ & $\mathrm{~B}$ & $\mathrm{C}$ & $\mathrm{D}$ & $\mathrm{E}$ & $\mathrm{F}$ \\
\hline & $2 / 5$ & $3 / 8$ & $4 / 5$ & $5 / 8$ & $6 / 5$ & $9 / 8$ \\
\hline$N_{s}$ & 12 & 9 & 24 & 15 & 36 & 27 \\
\hline Poles & 10 & 8 & 10 & 8 & 10 & 8 \\
\hline$a b+c$ & 2 & 3 & 4 & 5 & 6 & 9 \\
\hline Coil Through & \multicolumn{2}{|l|}{$\# 1 \sim \# 2$} & \multicolumn{2}{|l|}{$\# 1 \sim \# 3$} & \multicolumn{2}{|l|}{$\# 1 \sim \# 4$} \\
\hline$K_{w}$ & 0.933 & 0.945 & 0.925 & 0.952 & 0.924 & 0.941 \\
\hline$K_{p}$ & 0.966 & 0.985 & 0.966 & 0.995 & 0.966 & 0.985 \\
\hline$K_{d}$ & 0.966 & 0.960 & 0.958 & 0.957 & 0.956 & 0.955 \\
\hline
\end{tabular}

Each case can be designed the multi-pole design by less slots than ISW'. In this digest paper, we pick up some FSW methods from Table1, and described and explained in Slot Star Diagram. In this paper, we focused about the case of " $q<1 / 2$ " which is the concentrated winding.

\section{Winding Design Method using Slot Star DiagRam}

\section{Winding analysis used Slot Star Diagram}

Usually, when we calculate the winding factor in Table1, we used the formula (1). Alternatively, the method used the AC vectors in Slot Star Diagram. It showed the formula (2). (In this digest, the detailed description is omitted.). And alternatively, we know that it can be determined from Slot Star Diagram ${ }^{[2]}$. Fig. 2. shows the winding arrangement depended on Slot Star Diagram ${ }^{[2]}$. Fig.2 (a) is the diagram for $q=2 / 5$ whose number of slots is even, and Fig.2 (b) is the diagram for $q=3 / 8$ whose number of slots is odd.

$$
\begin{aligned}
& K_{w}=K_{p} K_{d}=\sin \left(r \frac{\pi}{2} \times \% \text { pitch }\right) \frac{\sin \left(r \times 30^{\circ}\right)}{(a b+c) \sin \left(r \times \frac{30^{\circ}}{a b+c}\right)} \\
& K_{w}(r)=\frac{\mathrm{AC} 1 \times \cos (r \cdot \theta 1)+\mathrm{AC} 2 \cos (r \cdot \theta 2)+\mathrm{AC} 3 \cos (r \cdot \theta 3) \cdots \cdots+\mathrm{AC} n \times \cos (r \theta n)}{\mathrm{ACl}+\mathrm{AC} 2+\mathrm{AC} 3 \cdot \cdot \cdot \mathrm{ACn}}
\end{aligned}
$$

Using the Slot Star Diagram of FSW which is concentrated winding and formula (2), we tried to calculate $K_{w}$ of 2 types. Formula (3) shows the calculation about $K_{w}$ of $q=2 / 5$ winding,

$$
K_{w}(r)=\frac{\mathrm{AC} 1 \times \cos (r \cdot 0)+2 \cdot \mathrm{AC} 2 \cos (r \cdot \pi / 6)}{\mathrm{AC} 1+2 \cdot \mathrm{AC} 2}
$$

Formula (4) shows the calculation about $K_{w}$ of $q=3 / 8$ winding,

$$
K_{w}(r)=\frac{2 \cdot \mathrm{AC} 1 \times \cos (r \cdot \pi / 18)+2 \cdot \mathrm{AC} 2 \cos (r \cdot \pi / 6)}{2 \cdot \mathrm{AC} 1+2 \cdot \mathrm{AC} 2}
$$



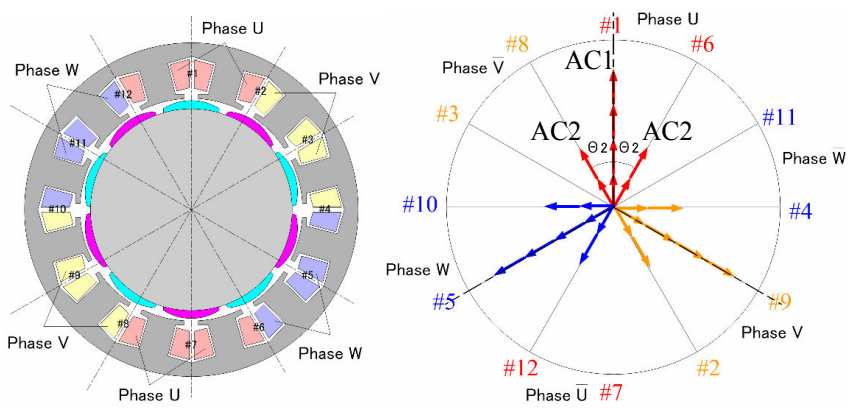

(a) FSW $q=2 / 5$ (10poles/12slots) depended on Slot Star Diagram
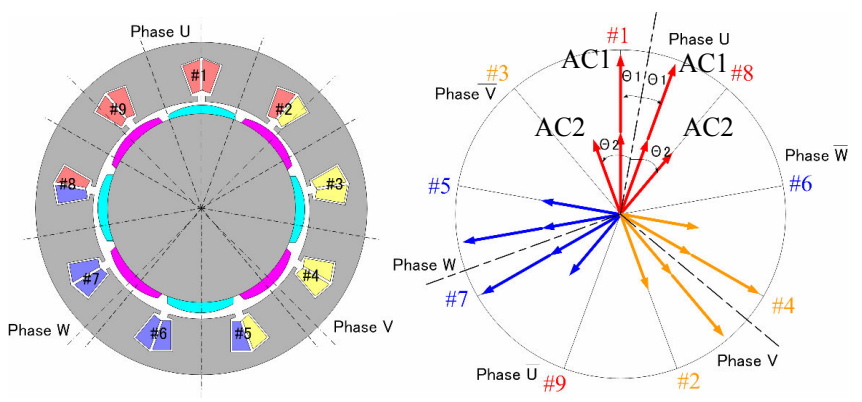

(b) FSW $q=3 / 8$ (8poles/9slots) depended on Slot Star Diagram

Fig.1. Winding arrangements of FSW $(q<1 / 2)$

Table2. Comparison of winding factor $K w$ of $q=2 / 5$ and $q=3 / 8$

\begin{tabular}{|c|c|c|c|}
\hline$K_{w}(r)$ & $r$ & $q=2 / 5$ & $q=3 / 8$ \\
\hline Fundamental & 1 & 0.933 & 0.945 \\
\hline $3^{\text {rd }}$ & 3 & 0.500 & 0.577 \\
\hline $5^{\text {th }}$ & 5 & 0.067 & 0.140 \\
\hline $7^{\text {th }}$ & 7 & 0.067 & -0.061 \\
\hline $9^{\text {th }}$ & 9 & 0.500 & 0.000 \\
\hline $11^{\text {th }}$ & 11 & 0.933 & 0.061 \\
\hline $13^{\text {th }}$ & 13 & 0.933 & -0.140 \\
\hline
\end{tabular}

These are the fundamental winding factor $K_{w}(1)$. Similarly, the harmonic winding factor $K_{w}(r)$ is shown at Table2. As shown in Fig.1, about comparison the distribution of the magnetic motive force (later shows MMF) vector at each slot between Fig1 (a) $q=2 / 5$ and (b) $q=3 / 8$, the $q=3 / 8$ is better than the $q=2 / 5$. Because, the MMF vector distributed at $q=3 / 8$ has more than the $q=2 / 5$. So about comparison the each harmonic winding factor $K_{w}(r)$, (b) $q=3 / 8$ is better than (a) $q=2 / 5$ too. Especially, about the high order harmonic winding factor like $K_{w}\left(11^{\text {th }}\right)$ and $K_{w}\left(13^{\text {th }}\right)$, the $q=3 / 8$ has a large advantage compared with the $q=2 / 5$.

From this consideration result, we can guess that the $q=3 / 8$ and the $q=2 / 5$ for the harmonics characteristics of EMF. Since, we describe for the both EMF characteristics with considering of the magnetic field.

\section{Magnetic field analysis}

As Table2 shows, in winding factor $K_{w}(r)$ the $q=2 / 5$ winding, especially, the $11^{\text {th }}$ and the $13^{\text {th }}$ harmonic winding factors are larger than the $q=3 / 8$. For the improvement of the harmonics characteristics in EMF, magnet shape that means magnetic field distribution, should be consider the optimum for each winding method.

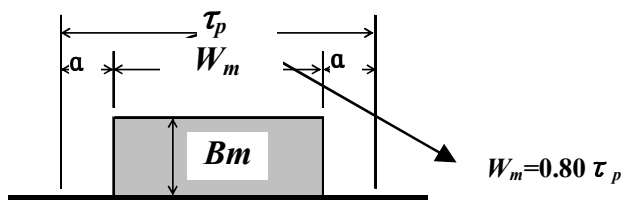

$\tau_{p}:$ Pole pitch, $W_{m}:$ Field Permanent Magnet Width $\alpha$ : Gaps between the magnets

Fig.2. Supposed magnetic filed form

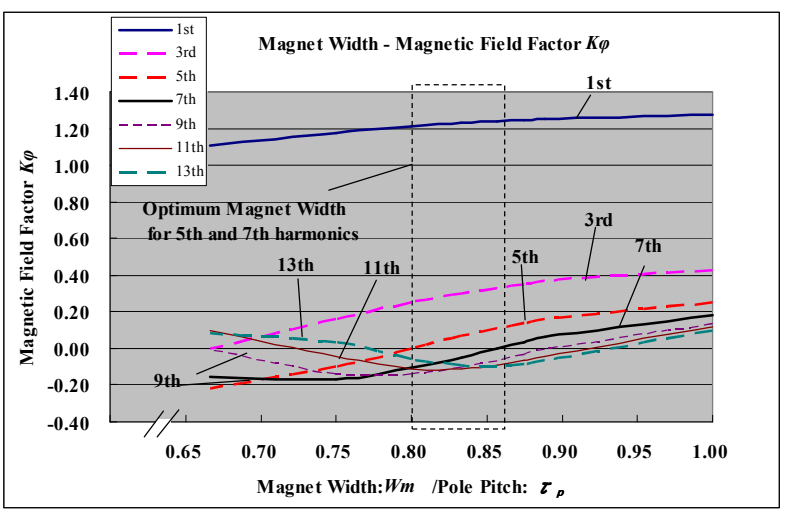

Fig.3. "Magnet width"- "Magnet flux factor $K_{\varphi} "$ graph

$$
K_{\varphi}(r)=\frac{4}{\pi}\left\{\frac{1}{r} \cos \left(r \frac{\alpha}{\tau_{p}} \pi\right)\right\}
$$

For, $\alpha=\left(\tau_{p}-W_{m}\right) / 2$

$r$ : Harmonics Order

For that, we should calculate each harmonic magnetic field factor $K_{\varphi}(r)$ of square magnetic field which block magnet. Formula (5) shows the Fourier analysis of square wave. On each harmonic magnetic field factor, it has the optimum magnet width $W_{m}$ which $K_{\varphi}$ should be " 0 ". So, we can be improve the harmonics characteristics of EMF by combined of the winding factor $K_{w}(r)$ and the magnetic field factor $K_{\varphi}(r)$.

\section{EMF analysis}

Formula (6) shows the calculation for EMF design. At the case of Star-connection of armature windings, the odd multiple of the $3^{\text {rd }}$ harmonic components in EMF is canceled, so we focused the $5^{\text {th }}$ and the $7^{\text {th }}$ components. The Low-order harmonic components should be canceled by the optimum magnet width. So in both the $q=2 / 5$ and the $q=3 / 8$, we should consider the optimum magnet width $W_{m}$ with the $5^{\text {th }}$ winding factor $K_{w}(5)$. As the result, " $4 / 5 \tau_{p}$ " is concluded optimal for the $5^{\text {th }}$ component in EMF at Fig.3.

$$
\begin{aligned}
& E(r)=\omega \cdot \Psi \\
& =\omega \cdot K_{w} \cdot \Phi_{m} \quad\left(\Phi_{m}=K_{\varphi} \cdot B_{m} \cdot S_{g}\right) \\
& =\omega \cdot K_{w} \cdot K_{\varphi} \cdot B_{m} \cdot S_{g} \\
& =\omega\left\{K_{w}(r) \cdot K_{\varphi}(r)\right\} B_{m} \cdot S_{g} \\
& \Psi \text { : Flux linkage }(\mathrm{Wb}), \quad \omega: \text { Rotational speed }(\mathrm{rad} / \mathrm{s}) \\
& \Phi_{m} \text { : Magnet flux }(\mathrm{Wb}), \quad B_{m} \text { : Magnet flux density (T) } \\
& S_{g} \text { : Gap flux area }\left(\mathrm{m}^{2}\right), \quad K_{\varphi}(r) \text { : Magnetic field factor }
\end{aligned}
$$


Table3. Comparison of harmonic components in EMF of $q=2 / 5$ and $q=3 / 8$

\begin{tabular}{|l|lr|l|}
\hline $\operatorname{EMF}(r)$ & $\begin{array}{l}q=2 / 5 \\
\left(\text { with } W_{m}=4 / 5 \tau_{p}\right)\end{array}$ & $\begin{array}{l}q=3 / 8 \\
\left(\text { with } W_{m}=4 / 5 \tau_{p}\right)\end{array}$ \\
\hline Fundamental (p.u.) & $\mathbf{1 . 0 0}$ & 1.01 \\
\hline $3^{\text {rd }}$ (p.u.) & 0.11 & 0.13 \\
\hline $5^{\text {th }}$ (p.u.) & 0.00 & 0.00 \\
\hline $7^{\text {th }}$ (p.u.) & -0.01 & 0.01 \\
\hline $9^{\text {th }}$ (p.u.) & -0.06 & 0.00 \\
\hline $\mathbf{1 1}^{\text {th }}$ (p.u.) & $\mathbf{- 0 . 0 9}$ & $\mathbf{- 0 . 0 1}$ \\
\hline $\mathbf{1 3}^{\text {th }}$ (p.u.) & $\mathbf{- 0 . 0 5}$ & $\mathbf{0 . 0 1}$ \\
\hline
\end{tabular}

Table 3 shows the comparison of harmonic components in EMF of the $q=2 / 5$ and the $q=3 / 8$ winding with $4 / 5 \tau_{p}$ magnet width. Looking at this, the $q=3 / 8$ is better than the $q=2 / 5$ for the $11^{\text {th }}$ and the $13^{\text {th }}$ components, at the harmonics characteristics of EMF.

The $q=3 / 8$ has the advantage for EMF characteristics. However at the case of mechanical and magnetic unbalance it has, it may also generate the vibrations. So, we considered the improvement method to reduce the $11^{\text {th }}$ and the $13^{\text {th }}$ harmonic components at the $q=2 / 5$, to level of the $q=3 / 8$ winding. And it used the irregular pitch winding method. Later, we will explain this.

\section{CONSIDERING ABOUT IRREgUlar PITCH Winding}

The irregular pitch method is for High-order harmonic component of EMF. Because, if it tried to reduce Low- order harmonics, the irregular pitch angle is too large. So realistically, its implementation is impossible. And for the irregular pitch winding, the even number of slot is possible only, because at the case of the even is symmetry at the Slot Star Diagram. But at the case of the odd, it is not line symmetry.

After we design the parameter about the irregular pitch angle to reduce the $11^{\text {th }}$ and $13^{\text {th }}$ component in EMF, we derived that the optimum irregular pitch electrical angle is $\pi / 22[\mathrm{rad}] \quad(8.13$ elec.deg). Fig.4 (a) shows the Slot Star Diagram for at the $q=2 / 5$ winding used irregular pitch angle $\pi / 22$ [rad] (8.13 elec.deg). And Fig.4 (b) shows the crosssectional view of PMSM at the $q=2 / 5$ winding described the irregular pitch mechanical angle $\pi / 110[\mathrm{rad}]$ (1.63mecha.deg).

【 $K_{w}(r):$ Irregular pitch $\theta_{i r}=\pi / 22[\mathrm{rad}](8.13$ elec.deg $) 】$

$K_{w}(r)=\frac{\mathrm{AC} 1 \times \cos (r \cdot \pi / 22)+\mathrm{AC} 2 \cos \{r \cdot(\pi / 6+\pi / 22)\}+A C 2 \cos \{r \cdot(\pi / 6-\pi / 22)\}}{\mathrm{ACl}+2 \cdot \mathrm{AC} 2}$

Table4. Comparison of harmonic components in EMF of regular pitch winding and irregular pitch winding at $q=2 / 5$

\begin{tabular}{|l|c|c|c|}
\hline \multirow{5}{*}{ EMF(r) } & \multicolumn{3}{|c|}{$\begin{array}{c}q=2 / 5 \\
\text { (with } W_{m}=4 / 5 \tau_{p} \text { ) }\end{array}$} \\
\cline { 2 - 4 } & \multicolumn{2}{|c|}{$\begin{array}{c}\text { Regular Pitch } \\
\theta_{i r}=0 \text { deg }\end{array}$} & $\begin{array}{c}\text { Irregular Pitch } \\
\theta_{i r}=\pi / 22[\mathrm{rad}]\end{array}$ \\
\cline { 2 - 4 } & Data-A & Data-B & Data-C \\
\cline { 2 - 4 } & $\begin{array}{l}\text { Fig.5 (a) } \\
\text { Block Mag. }\end{array}$ & $\begin{array}{c}\text { Fig.5 (b) } \\
\text { Arch Mag. }\end{array}$ & $\begin{array}{c}\text { Fig.5 (a) } \\
\text { Block Mag. }\end{array}$ \\
\hline Fundamental (p.u.) & $\mathbf{1 . 0 0}$ & $\mathbf{0 . 9 0}$ & $\mathbf{0 . 9 9}$ \\
\hline $3^{\text {rd }}$ (p.u.) & 0.11 & 0.04 & 0.10 \\
\hline $5^{\text {th }}$ (p.u.) & 0.00 & 0.00 & 0.00 \\
\hline $7^{\text {th }}$ (p.u.) & -0.01 & 0.00 & 0.00 \\
\hline $9^{\text {th }}$ (p.u.) & -0.06 & -0.03 & -0.02 \\
\hline $\mathbf{1 1}^{\text {th }}$ (p.u.) & $\mathbf{- 0 . 0 9}$ & $\mathbf{- 0 . 0 0}$ & $\mathbf{0 . 0 0}$ \\
\hline $\mathbf{1 3}^{\text {th }}$ (p.u.) & $\mathbf{- 0 . 0 5}$ & $\mathbf{- 0 . 0 1}$ & $\mathbf{0 . 0 1}$ \\
\hline
\end{tabular}

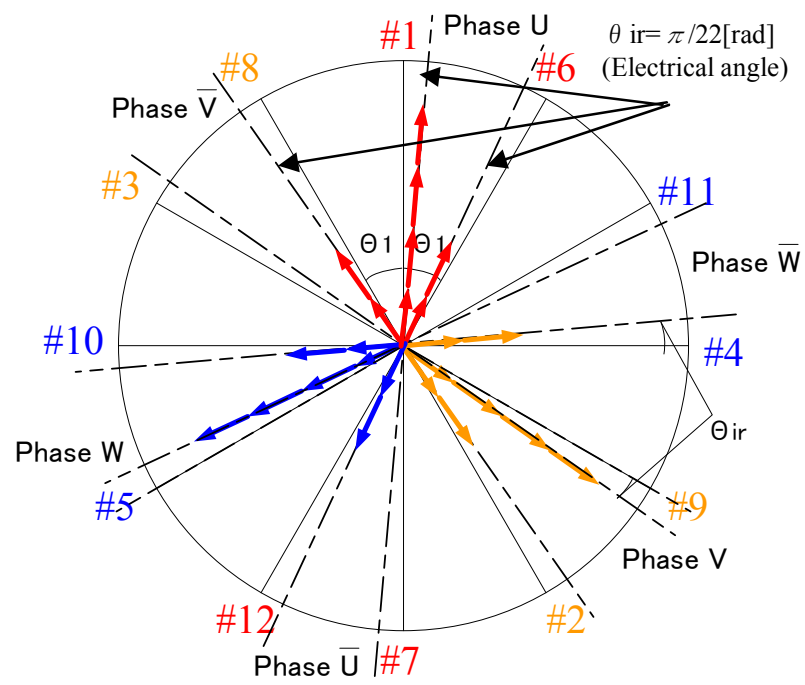

(a) Slot Star Diagram for irregular pitch winding at $q=2 / 5$ Irregular pitch angle $\theta_{i r}=8.25$ (electrical degree)

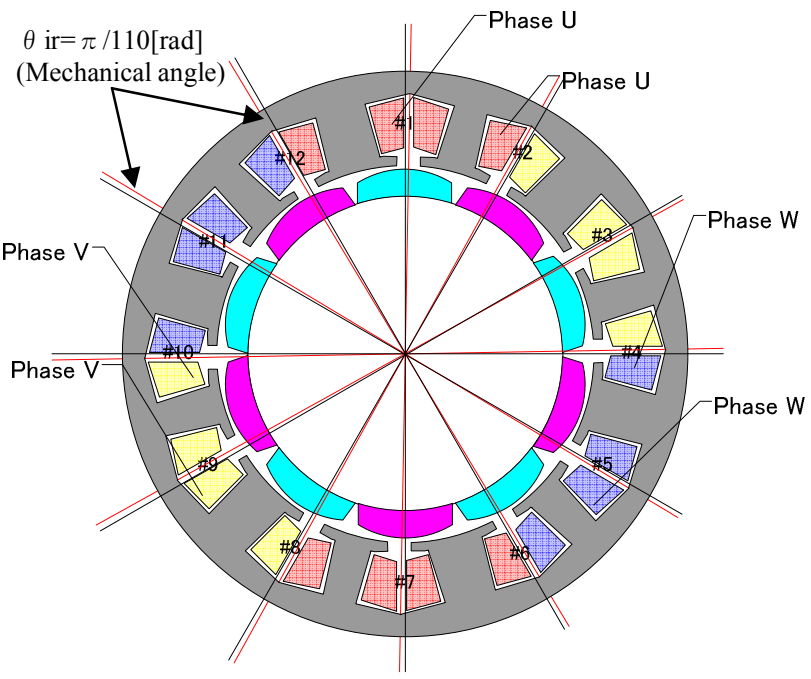

(b) Cross-sectional view of PMSM used irregular pitch winding at $q=2 / 5$ Irregular pitch angle $\theta_{i r}=1.65$ (Mechanical degree)

Fig.4. Irregular pitch winding method at $q=2 / 5$

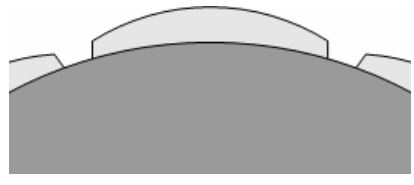

(a) Arch magnet

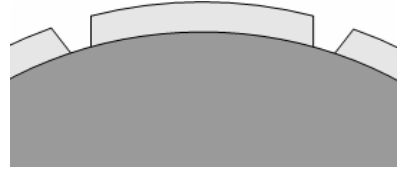

(b) Block magnet
Fig.5 Magnet shape considered for EMF

Looking at this, the armature has two type teeth, one is wide and another is thin by performing the irregular pitch winding. Fig.5 shows two type the magnet shape, (a) is arch shape produce close to sine wave, and (b) is block shape produced square wave magnetic filed. This arch magnet is usually used at the case of the regular pitch winding to reduce harmonic components in EMF.

Formula (7) shows is the calculation for this irregular pitch's, and Table4 shows is the calculation result about harmonic components in EMF at the irregular pitch used this 
formula. In Table4, Data-A is the result with block magnet shape shown at Fig.5 (a), and data-B is the result with arch magnet shape shown at Fig.(b). This has known from traditional, as one of methods to improve EMF wave form. Data-C is the result with block magnet shape shown Fig.(a).

Looking at this result, the irregular pith method can reduce the $11^{\text {th }}$ and $13^{\text {th }}$ harmonic in EMF well without reduction the fundamental component. In addition as mentioned earlier, the arch magnet is usually use at the regular pitch method to reduce harmonic components. So, the effect of this irregular pitch method is same level compared with the case used the arch magnet, and it can be $10 \%$ higher than the case of arch magnet at the motor constant $K_{m}$.

\section{ADVANTAGES OF IRREGULAR PITCH WINDING METHOD}

We summarized as follows the advantages about the irregular pitch winding method.

1) Higher motor constant $K_{\underline{m}}$ :

This irregular pitch method has the higher motor constant compared with conventional regular pitch method used arch magnet. So it can produce the effect the improvement for acceleration characteristics.

2) Saving Dysprosium (Later shows $D y$ ) in Nd-Fe-B magnet:

Fig.6 shows the comparison of electro-magnetic construction for sinusoidal EMF about regular pitch winding method and irregular pitch winding method. Looking at Fig.6 (a) used arch magnet, when the armature reaction is applied to the field magnet, the magnet edge is affected demagnetizing most by this armature reaction.

The edge of arch magnet thickness is about half of the block magnet's at same position. And, the arch magnet is easy to demagnetization than the block magnet. Therefore, at the case of using the arch magnet, it is necessary to use a favorable temperature characteristics used much $D y$ content. However, this irregular pitch method with the optimized magnet width is possible to use block magnet. So the magnet thickness is thick and it is be strong to demagnetization. From this, it can be used the magnet whose $D y$ content is half quantity compared with the case of arch magnet.

\section{CONCLUSION}

In this paper, we report the improvement effect for EMF characteristics used the irregular pitch winding method at $q=2 / 5$, and we found the possibility about efficiency and $D y$ saving for Nd-Fe-B magnet. Especially, the harmonic components of EMF at the $q=2 / 5$, can be reduce to same level of the $q=3 / 8$ winding. So it has the excellent torque ripple performance. However, it is considering design only in this paper. We should inspect the effect which this irregular pitch winding method gives to cogging and vibration performance.

Future, we will study of validity of this idea used FEM simulation and evaluation by the actual equipment.

\section{REFERENCES}

[1] Johannes Klamt : “Berechnung und electrisher Maschunen”, produced by Springer-Verlag pp.192-198 (1962)

[2] Yasuhiro Miyamoto, Tsuyoshi Higuchi, Takashi Abe: "Consideration for Fractional Slot Winding of Permanent Magnet Type Synchronous Machine”, ICEMS-2011 Collection of paper-14 (2011-Beijing Chaina)

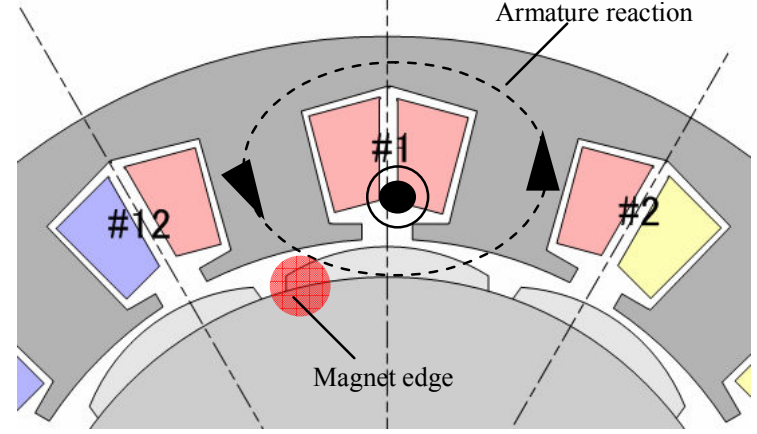

(a) Armature construction used regular pitch winding for sinusoidal EMF

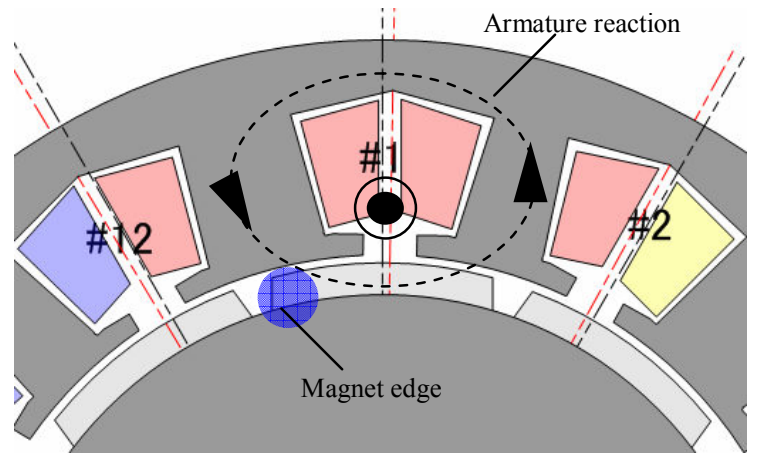

(b) Armature construction used irregular pitch winding for sinusoidal EMF

Fig.6. Comparison of electro-magnetic construction for sinusoidal EMF between regular pitch winding and irregular pitch winding method

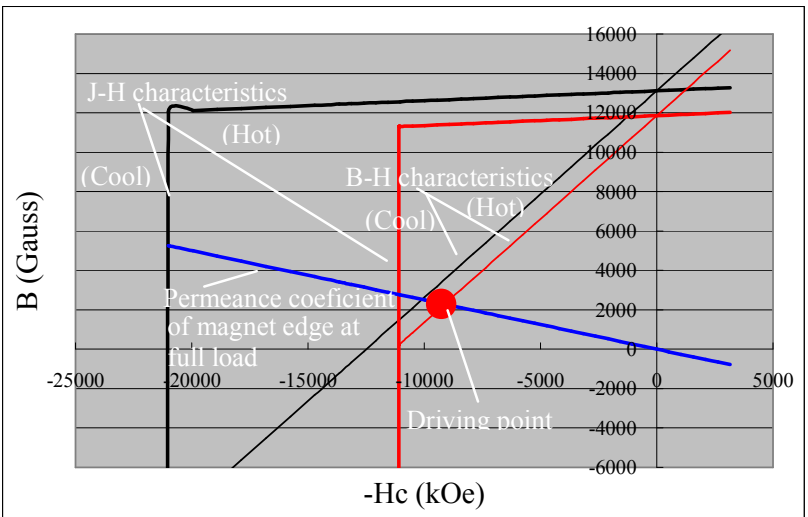

(a) Driving point of arch magnet with much $D y$ content $\left(\mathrm{H}_{\mathrm{cj}}=21 \mathrm{kOe}\right)$

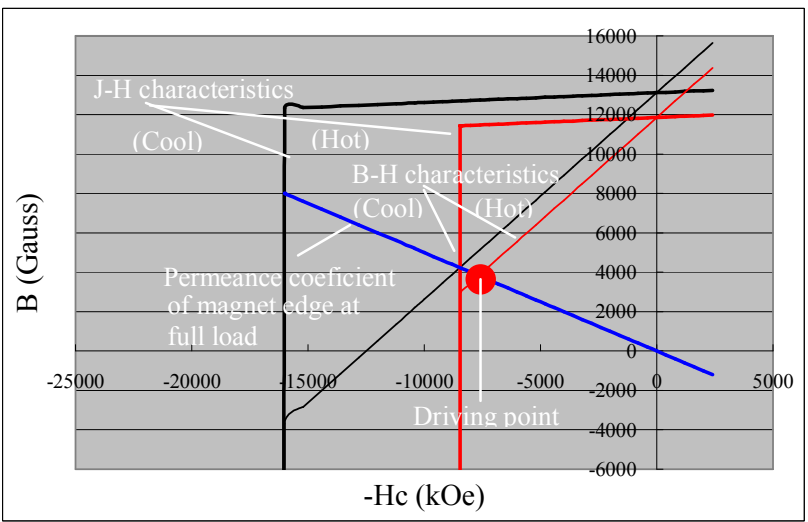

(b) Driving point of block magnet with much $D y$ content $\left(\mathrm{H}_{\mathrm{cj}}=16 \mathrm{kOe}\right)$

Fig.7 Permeance coefficient $k_{p}$ and driving point on thermal characteristics of $\mathrm{Nd}-\mathrm{Fe} \mathrm{B}$ magnet 